\title{
Evolution saisonnière de la physico-chimie et du phytoplancton dans la retenue de Pareloup sur une longue période (1983-1998) : incidence d'une vidange totale
}

\author{
J.-M. Thébault ${ }^{1}$
}

Mots-clés : phytoplancton, cycles physico-chimiques, réservoir, vidange, diatomées, Asterionella formosa, Aulacoseira.

La retenue de Pareloup a été étudiée depuis 1983. En 1993, elle a été entièrement vidangée ce qui a permis d'étudier l'effet de cette perturbation sur l'écosystème. Ce papier fait la synthèse de l'ensemble des données recueillies depuis le début de l'étude, et décrit l'évolution avant et après la vidange, des variables physico-chimiques et du peuplement phytoplanctonique. Un suivi de 16 ans permet de s'affranchir des années exceptionnelles et de voir à long terme comment les conditions environnementales et l'écosystème évoluent. Les concentrations de nitrates ont augmenté en 1986 dans l'ensemble du bassin versant et dans la retenue. Elles sont en légère augmentation, ou stabilisées selon les entrées, les années suivantes. L'accroissement des concentrations de silice et de nitrates observées dans la retenue après la vidange ont pour origine l'augmentation des apports liée à trois années pluvieuses et non la vidange elle-même. Le fonctionnement de l'écosystème a été fortement perturbé l'année qui a suivit la vidange : disparition des espèces dominantes du phytoplancton et développement d'espèces jusqu'alors peu abondantes. L'écosystème a ensuite retrouvé son cycle initial dès 1995. La diminution du temps de séjour semble favoriser le développement des diatomées centrales (Aulacoseira sp.) au détriment d'Asterionella formosa. Compte tenu du rôle d'A. formosa dans le piégeage du phosphore par sédimentation, la dominance d'Aulacoseira certaines années pourrait perturber l'évolution estivale de la retenue.

Seasonal evolution of the physico-chemistry and the phytoplankton of Pareloup reservoir over a long period (1983-1998): impact of total draining

Keywords : phytoplankton, physico-chemical cycles, dammed lake, draining, diatoms, Asterionella formosa, Aulacoseira.

Lake Pareloup has been studied since 1983. In 1993 it was completely drained, providing an opportunity to study the effect of this disturbance on the ecosystem. This paper gives a synthesis of all data collected since the beginning of the study. and describes the physico-chemical variables and phytoplankton population before, and subsequent to, drainage. The results of monitoring over a 16 year period provide a long-term overview of environmental conditions and ecosystem development, compensating for undue disturbance in data from exceptional years. Nitrate concentrations increased slightly in 1986 in the watershed and in the lake, but stabilised in the following years. The observed post-drainage increased concentrations of silica and nitrates in the lake is attributed to increased inflow during three years of exceptional rainfall and not to the drainage itself. Ecosystem function was strongly disturbed in the year following drainage, with the replacement of the dominant phytoplanktonic species by species previously not very abundant. The ecosystem reverted to its original condition from 1995. A reduction in residence time seems to favour the development of centric diatoms (Aulacoseira sp) to the detriment of $A$. formosa. Considering the role of $A$. formosa in trapping phosphorus by sedimentation, the dominance of Aulacoseira in some years could disrupt the summer seasonal evolution of the ecosystem.

1. Centre d'Ecologie des Systèmes Aquatiques Continentaux, UMR C5567, Université Paul Sabatier, 118 route de Narbonne, 31062 Toulouse Cedex 04, France.

E-mail : thebault@cict.fr 


\section{Introduction}

La retenue de Pareloup a été étudiée depuis 1983 a des fins de connaissance scientifique, ce qui a fait l'objet d'une convention de 4 ans (1986-1990) entre Electricité de France (EDF) et le Ministère de l'Environnement représenté par le Laboratoire d'Hydrobiologie de Toulouse (actuel CESAC : Centre d'Ecologie des Systèmes Aquatiques Continentaux).

Le réservoir de Pareloup a été vidangé en juin 1993. Cet événement en a fait un site idéal pour étudier l'effet d'une telle perturbation sur un écosystème lacustre à l'échelle de plusieurs années. Aussi, afin de comparer le fonctionnement de l'écosystème entre les dernières années précédant la vidange et les premières années suivant le remplissage, un programme d'étude et de recherche a été établi entre le Département Environnement de la DER d'EDF et le CESAC.

Ce papier fait le point sur l'ensemble des mesures qui ont été effectuées, dépouillées et traitées, sur la retenue de Pareloup et ses affluents depuis 1983 (depuis 1976 pour les débits). La physico-chimie de la retenue de Pont de Salars, mesurée depuis 1993, sera brièvement décrite car ses eaux alimentent Pareloup par la station de pompage de Bage.

\section{Description de la retenue de Pareloup}

La retenue de Pareloup est située dans le département de l'Aveyron, au sud-ouest du Massif Central (France). La superficie totale du bassin versant est de $168 \mathrm{~km}^{2}$, dont les trois quarts sont destinés à l'élevage. Le lac de Pareloup est le réservoir culminant du complexe hydroélectrique du Pouget. La très grande capacité de cette retenue permet d'emmagasiner l'eau pendant les saisons de moindre besoin en électricité et de l'utiliser pendant les saisons de forte demande (principalement l'hiver). La cote du plan d'eau reste haute et stable en été, pendant la période touristique.

Outre les apports naturels du Vioulou, le principal affluent, et d'autres petits ruisseaux, la retenue reçoit l'eau des bassins versants du Viaur et du Bage, par l'intermédiaire des retenues de Pont de Salars $\left(20 \mathrm{hm}^{3}\right)$ et de Bage $\left(4 \mathrm{hm}^{3}\right)$, qui fonctionnent en vases communicants. La station de pompage de Bage (débit maximum $14 \mathrm{~m}^{3} \cdot \mathrm{s}^{-1}$ ), remonte ces apports sur les $98 \mathrm{~m}$ de dénivellation séparant Bage de Pareloup, avec un débit annuel moyen de $4,21 \mathrm{~m}^{3} \cdot \mathrm{s}^{-1}$. La conduite de Bage débouche dans le fond du lac à la cote 768 NGF (Niveau Général de la France).

L'eau stockée dans Pareloup est turbinée à l'usine d'Alrance (chute de $80 \mathrm{~m}$, débit maximum $24 \mathrm{~m}^{3} \cdot \mathrm{s}^{-1}$ ) puis, transite par le réservoir de Villefranche de Panat $\left(11 \mathrm{hm}^{3}\right)$, et est restituée au Tarn à l'usine du Pouget qui exploite une chute de $461 \mathrm{~m}$. Le lac est composé d'un corps principal orienté est-ouest correspondant à la vallée du Vioulou sur lequel est construit le barrage et de bras latéraux.

A la cote maximale (805 NGF), sa capacité totale est de $169 \mathrm{hm}^{3}$ et la superficie de $12,6 \mathrm{~km}^{2}$. En raison de sa forme très digitée, sa longueur de rive est considérable : $120 \mathrm{~km}$. La profondeur maximale de la retenue à proximité du barrage est de 40 mètres. Elle diminue rapidement à 25 mètres au droit de l'ancien lit du Vioulou. L'essentiel du volume de la retenue est situé en surface, la capacité utile entre les cotes 775 et 805 NGF représentant $167,7 \mathrm{hm}^{3}$. La profondeur moyenne est de $12,5 \mathrm{~m}$ et le centre de volume du lac est situé à $10 \mathrm{~m}$.

Les sorties d'eau de Pareloup sont de deux sortes : le débit réservé du Vioulou, restitué par une vanne en pied de barrage, et le débit turbiné. Le débit réservé, prélevé à la cote $764 \mathrm{NGF}$, est faible $\left(1031 . \mathrm{s}^{-1}\right)$, alors que le débit turbiné prélevé dans le lac par une tour dont l'ouverture est comprise entre les cotes 775 NGF et $784 \mathrm{NGF}$, peut atteindre $24 \mathrm{~m}^{3} \cdot \mathrm{s}^{-1}$ (EDF 1994, Salençon \& Thébault 1997).

La retenue a été entièrement vidée en 1993 afin d'effectuer une inspection réglementaire et des travaux d'entretien. L'évacuation s'est faite progressivement par turbinage, puis par le cours du Vioulou. On peut distinguer quatre phases : l'abaissement (novembre 1992-juin 1993), la vidange proprement dite (ouverture de la vanne de fond du 14 au 17 juin), la phase d' «àsec» jusqu'au 17 septembre et la remise en eau jusqu'en mars 1994 (Merle et al. 1994).

\section{Les apports du bassin versant et l'hy- draulique}

\subsection{Traitement des données disponibles}

Les données disponibles sont présentées dans le tableau 1. Les données manquantes de débit du Rieutord et du Connes ont été reconstituées à partir du débit du Vioulou et de la surface de leurs bassins versants respectifs. La comparaison des débits mesurés et estimés de cette façon montre une excellente concordance. De la même manière, les apports d'eau de l'ensemble du bassin versant ont été calculés à partir des débits du Vioulou et de la surface totale du bassin versant $\left(168 \mathrm{~km}^{2}\right)$.

Pour les nutriments, les années qui ont pu faire l'objet d'une analyse sont celles pour lesquelles nous dis- 
Tableau 1. Données de débit et de chimie disponibles pour les affluents, le pompage et le turbinage.

Table 1. Available data of flow rates and chemistry for affluents, pumping and turbining.

\begin{tabular}{ccccccc}
\hline & Vioulou & Rieutord & Connes & Bage & Alrance & Viaur \\
\hline Débit & $76-98$ & $6 / 83-5 / 85 ; 7 / 86-8 / 87$ & $6 / 83-5 / 85 ; 7 / 86-8 / 87$ & $76-98$ & $76-98$ & $76-98$ \\
$\mathrm{MES}$ & $83-87 ; 95-98$ & $83-87 ; 95-98$ & $83-87 ; 95-98$ & $83-87 ; 94-98$ & $95-96 ; 98$ & $94-98$ \\
$\mathrm{~N}-\mathrm{NO}_{3}$ & $83-87 ; 95-98$ & $83-87 ; 95-98$ & $83-87 ; 95-98$ & $83-87 ; 94-98$ & $95-96 ; 98$ & $94-98$ \\
$\mathrm{~N}-\mathrm{NH}_{4}$ & $87 ; 95-96$ & $87 ; 95-96$ & $87 ; 95-96$ & $87 ; 94-96$ & $95-96 ; 98$ & $94-96$ \\
$\mathrm{P}_{-} \mathrm{PO}_{4}$ & $86-87 ; 95-98$ & $83-87 ; 95-98$ & $83-87 ; 95-98$ & $83-87 ; 94-98$ & $95-96 ; 98$ & $94-98$ \\
$\mathrm{P}$ total & $83-87$ & - & - & - & $86-87$ & - \\
\hline
\end{tabular}

posons de mesures représentatives de l'ensemble de l'année, hebdomadaires en général. Les mesures de $\mathrm{NH}_{4}$ présentant de nombreux «trous» (mauvaise conservation des échantillons due à des dosages tardifs), seules trois années $(1987,1995$ et 1996) ont été retenues. Le P total n'a plus été mesuré après 1987 . Le transport du phosphore dans les eaux superficielles étant étroitement associé à celui des MES, nous avons pu reconstituer la chronologie de $\mathrm{P}$ total sur la période 1995-1998 en établissant une relation entre $P_{\text {tot }}$, MES et $\mathrm{P}-\mathrm{PO}_{4}$ mesurés simultanément :

$$
\mathrm{PO}_{4} / \mathrm{Ptot}=1.2643 . \mathrm{MES}^{-0.467} ; \mathrm{r}^{2}=0.723
$$

\subsection{Le bassin versant de Pareloup}

La figure 1a présente les débits moyens annuels de 1976 à 1998, pour d'une part, les deux sources d'entrée, les apports estimés du bassin versant et le pompage à Bage et, d'autre part, la sortie par turbinage d'Alrance (le débit réservé est négligeable). A l'échelle annuelle, les entrées naturelles et les entrées par pompage sont quasiment équivalentes. 1989, 1990 et 1991 sont les années les plus sèches depuis le début des mesures en $1976 ; 1994$ et 1996 sont des années exceptionnellement pluvieuses. Il s'ensuit de grosses différences inter-annuelles du temps de séjour, allant de 596 jours en 1990 à 189 jours en 1996 (Fig. 1b). Nous verrons l'importance de ces années pluvieuses sur les apports de nutriments.

Pour chaque entrée, les concentrations en nutriments et les flux annuels ont été calculés de la façon suivante :

Concentration moyenne annuelle $=\Sigma C_{i} Q_{i} / \Sigma Q_{i}$

Flux moyen annuel $=\left(\Sigma \mathrm{C}_{\mathrm{i}} \mathrm{Q}_{\mathrm{i}} / \Sigma \mathrm{Q}_{\mathrm{i}}\right) *$ volume annuel
$\mathrm{Ci}=$ concentration mesurée au jour $\mathrm{i}, \mathrm{Qi}=$ débit moyen journalier le jour de la mesure, volume annuel = somme des débits journaliers.

Cette méthode (Moatar \& Meybek 2001) permet de filtrer l'hydrologie, surtout lorsque la concentration ponctuelle est indépendante du débit (cas du $\mathrm{P}$ en général).

Pour l'estimation des flux de nutriments provenant de l'ensemble du bassin versant, nous avons exclu la surface du miroir et traité à part le bassin du Connes dont la charge est plus élevée que celle du reste du bassin du versant :

$$
\text { flux } B V=f_{C}+\left[\left(f_{V}+f_{R}\right) \cdot\left(s_{B V}-s_{M}-s_{C}\right)\right] /\left(s_{V}+s_{R}\right)
$$

$f_{C}, f_{V}, f_{R}=$ flux d'apport dans le Connes, le Vioulou et le Rieutord

$s_{B V}, s_{C}, s_{V}, s R, s_{M}=$ surface du bassin versant total, du Connes, du Vioulou, du Rieutord et surface du lac.

Les concentrations en nitrates des affluents ont fortement augmenté en 1986 (Salençon et al. 1989, Sivadier et al. 1994) puis ont continué leur progression plus ou moins marquée jusqu'en 1997. L'augmentation à Bage est plus régulière et se stabilise dès 1996. La valeur des concentrations en $\mathrm{NO}_{3}$ est plus ou moins identique pour chacune des quatre entrées (Fig. 2). La concentration de $\mathrm{NH}_{4}$ a considérablement diminué depuis 1987. La proportion par rapport au N-NO de 10 à $15 \%$ en 1987 à moins de $1 \%$ en 1996 (Tableau 2). Les concentrations de $P$ total sont plus élevées que la moyenne dans le Vioulou de 1985 à 1987, puis reviennent ensuite aux valeurs initiales faibles $(<50$ $\left.\mu \mathrm{g} . \mathrm{l}^{-1}\right)$; les concentrations dans le Rieutord sont également faibles, ce qui, compte tenu des concentrations en 

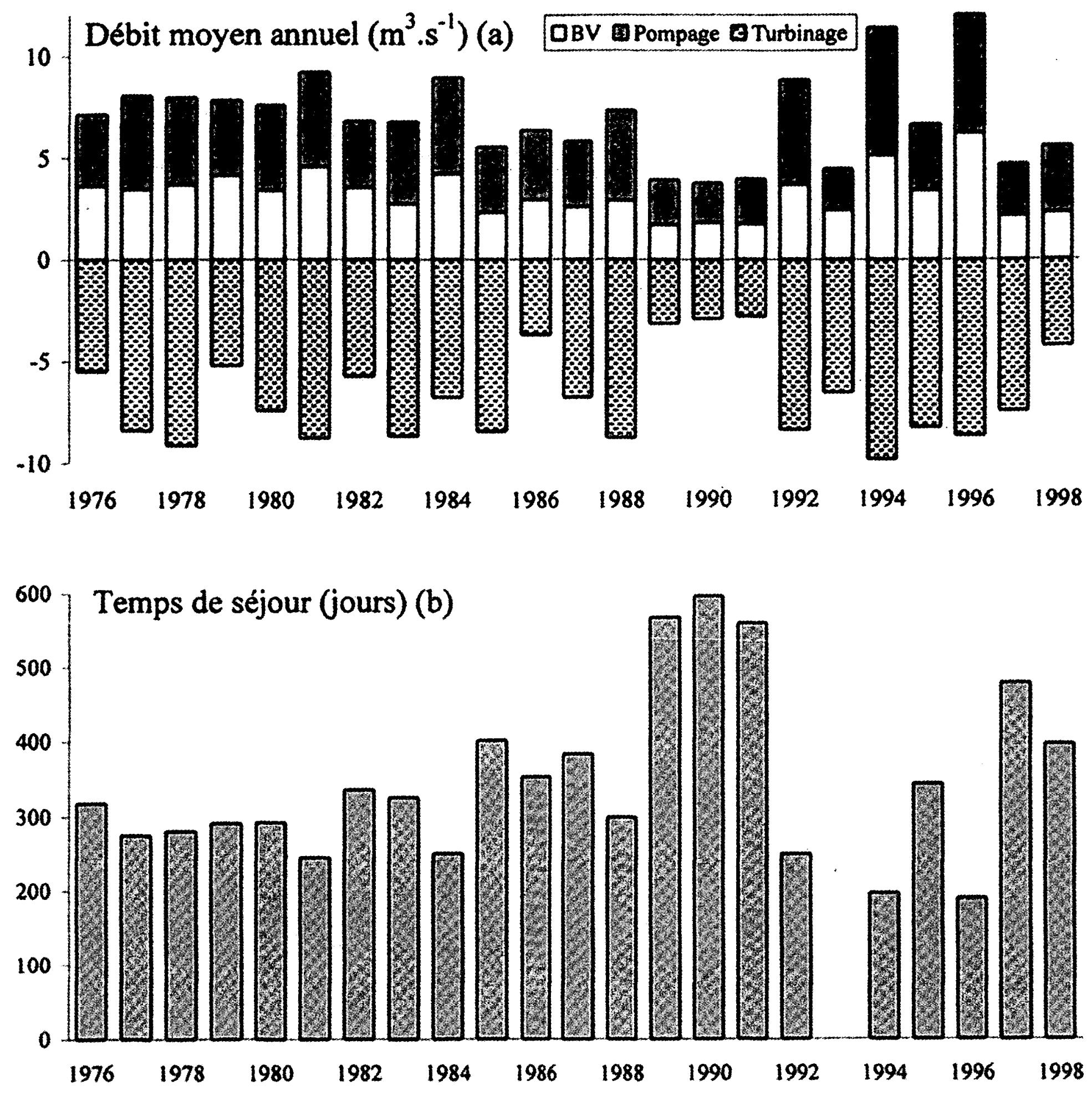

Fig. 1. (a) Débit moyen annuel des entrées d'eau par le bassin versant et par le pompage à Bage et débit moyen de la sortie par turbinage à Alrance.

(b) Temps de séjour de la retenue de 1976 à 1998.

Fig. 1. (a) Annual average inflow of water from the watershed and the pumping from Bage and average outflow turbined to Alrance. (b) Residence time of the reservoir from 1976 to 1998. 
Vioulou
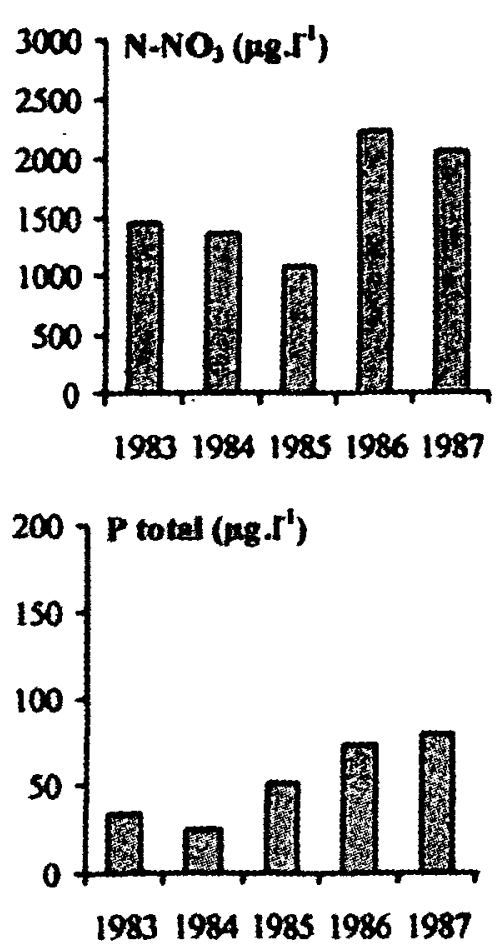

Rieutord
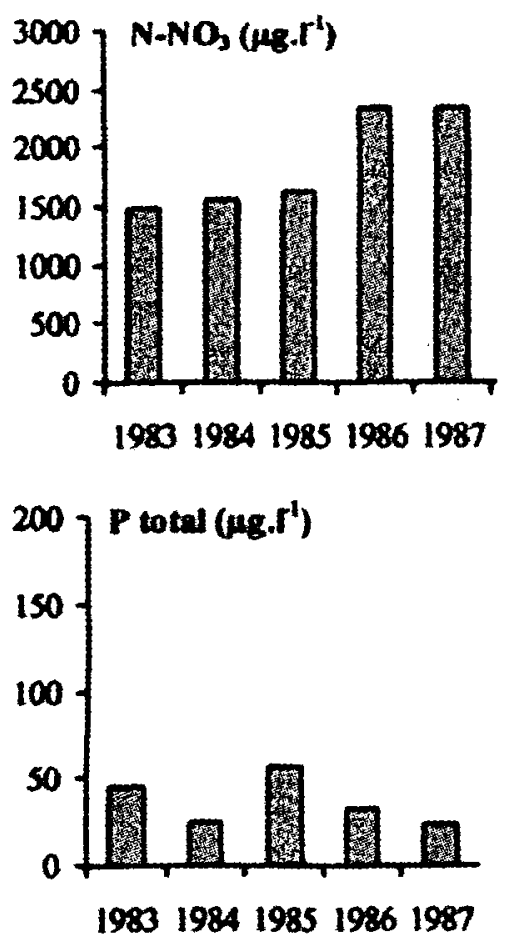
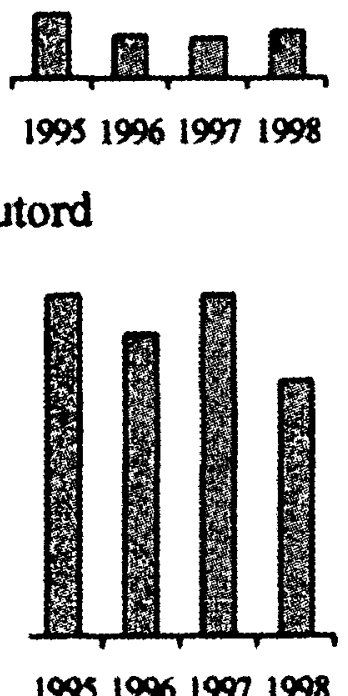

1995199619971998

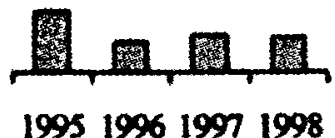

\section{Connes}
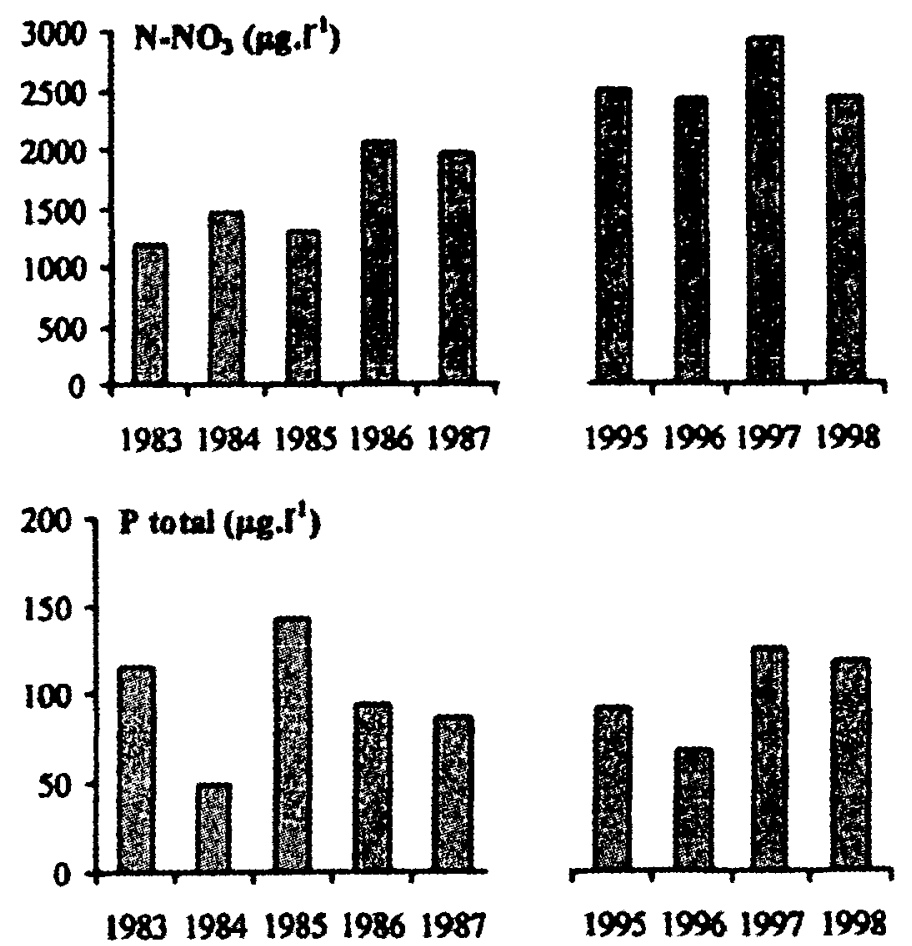

Bage
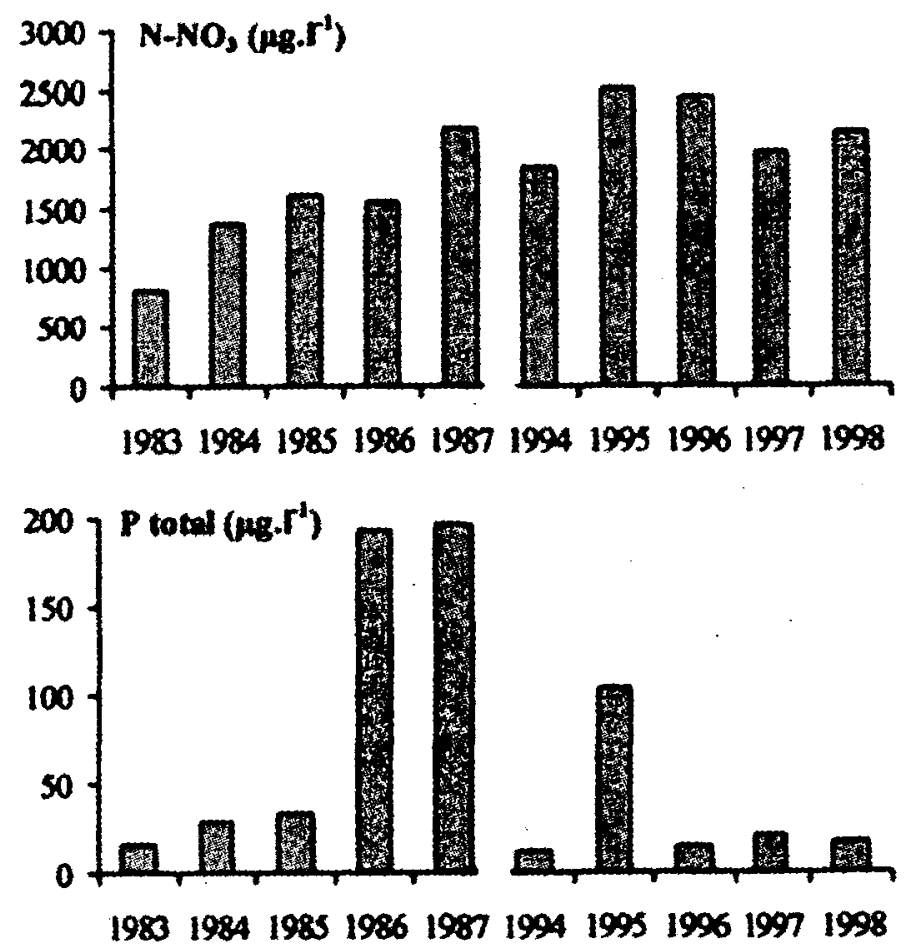

Fig. 2. Concentration moyenne de $\mathrm{N}-\mathrm{NO}_{3}$ et $\mathrm{P}$ total dans les affluents et dans l'eau pompée à Bage.

Fig. 2. Average concentration of $\mathrm{N}-\mathrm{NO}_{3}$ and total $\mathrm{P}$ in tributaries and in water pumped from $\mathrm{Bage}$. 
Tableau 2. Proportion de $\mathrm{N}-\mathrm{NH}_{4}$ par rapport au N-NO${ }_{3}$ dans les entrées.

Table 2. Proportion of $\mathrm{N}-\mathrm{NH}_{4}$ in relation to the $\mathrm{N}-\mathrm{NO}_{3}$ in the inflow.

\begin{tabular}{ccccc}
\hline & \multicolumn{4}{c}{$\%$ de $\mathrm{N}-\mathrm{NH}_{4}$ par rapport à $\mathrm{NO}_{3}$} \\
& Vioulou & Rieutord & Connes & Bage \\
\hline 1987 & $15 \%$ & $8.8 \%$ & $12.6 \%$ & $11 \%$ \\
1994 & - & - & - & $3 \%$ \\
1995 & $0.6 \%$ & $0.3 \%$ & $1.6 \%$ & $0.5 \%$ \\
1996 & $0.4 \%$ & $0.8 \%$ & $0.7 \%$ & $0.3 \%$ \\
\hline
\end{tabular}

$\mathrm{NO}_{3}$, est représentatif pour ces deux bassins versants d'une occupation des sols principalement agricole et peu peuplée ( 15 et 20 hab. $\mathrm{km}^{-2}$ ). Bien que le bassin du Connes ne soit pas plus peuplé, le ruisseau reçoit les eaux de la commune de Salles-Curan (1100 habitants permanents, beaucoup plus en été). Les concentrations varient d'une année à l'autre et peuvent atteindre $150 \mu \mathrm{g} . \mathrm{l}^{-1}$. Une station de lagunage ne fonctionnant que l'été, pour des raisons évidentes de température d'eau, a été construite en 1996 entre Salles-Curan et l'embouchure du Connes. Les concentrations de nitrates ne semblent pas cependant avoir été réduites depuis ; quant à celles de $\mathrm{P}$ total, elles ont augmenté après 1996. On peut cependant raisonnablement penser que les concentrations auraient été plus élevées en l'absence de station compte tenu du développement touristique de ce bassin versant. A Bage, les concentrations de $\mathrm{P}$ total sont peu élevées $\left(<50 \mu \mathrm{g} . \mathrm{l}^{-1}\right)$, excepté en 1986 et $1987\left(200 \mu \mathrm{g} . \mathrm{l}^{-1}\right)$ et, dans une moindre mesure, en 1995 ( $>100 \mu \mathrm{g} . \mathrm{l}^{-1}$ ). L'origine de ces augmentations, provenant du bassin versant du Viaur, n'est pas clairement définie.

Au niveau saisonnier, sur les trois affluents, les concentrations de $\mathrm{N}$ sont maximum en hiver et liées au débit. A l'inverse, les concentrations en $\mathrm{P}$ sont maximales en été et en automne, à cause de l'augmentation estivale de la population. L'amplitude de cet effet saisonnier est relativement stable, voire en légère diminu- tion à partir de 1995, pour les bassins du Vioulou et du Rieutord. En revanche, elle s'amplifie très nettement pour le bassin du Connes en 97 et 98 . Ceci est dû à une augmentation de la population estivale sur ce bassin versant (aménagement de campings, de gîtes, etc.)

Les flux (Fig. 3) : les apports de $\mathrm{N}_{-} \mathrm{NO}_{3}$ sont proportionnels aux débits des affluents et du pompage. La quantité de $\mathrm{N}-\mathrm{NO}_{3}$ apportée par Bage est équivalente ou un peu plus élevée que celle apportée par le bassin versant, selon la proportion de leur débit respectif. Comme l'avaient signalé Sivadier et al. (1994), les flux de P total ne sont pas liés aux débits. En effet, en période d'étiage, de très faibles variations de débit suffisent à mobiliser de grandes quantités de $\mathrm{P}$, accumulées dans le lit de la rivière. A l'inverse, en période de hautes eaux, les concentrations de $\mathrm{P}$ sont faibles. On constate des apports importants de P par Bage en 1986 et 1987, ainsi qu'en 1995. Cette augmentation s'observe également dans les apports du Vioulou, mais compte tenu des débits, les quantités sont beaucoup plus faibles. Les autres années, les apports par le bassin versant et Bage sont stables. Les apports de P par le Connes sont presque aussi élevés que ceux du Vioulou avec pourtant des débits de deux à trois fois plus faibles.

Nous disposons de trois années de mesures de $\mathrm{NO}_{3}$ et $\mathrm{P}$ total dans l'eau turbinée à Alrance (1995-1998: il n'y a pratiquement pas eu de turbinage en 1997), ce qui permet de faire le bilan du $\mathrm{N}$ et $\mathrm{P}$ stockés dans la retenue (Tableau 3). Environ $30 \%$ des nitrates sont stockés dans la retenue, quels que soient les flux d'entrée. Par contre, la proportion de $\mathrm{P}$ piégée par la retenue dépend de la quantité des apports. Plus la quantité de $\mathrm{P}$ apportée est élevée, plus la proportion de $\mathrm{P}$ stockée est importante. Sivadier et al. (1994), avaient calculé que $90 \%$ du P était stocké dans la retenue. Compte tenu des apports des années 1986-1987, ces résultats sont en accord avec une année comparable, 1995.

\subsection{Le bassin versant et la retenue de Pont de Sa- lars}

Des mesures de physico-chimie, de chlorophylle et des comptages du phytoplancton (Chung 2001) ont été

Tableau 3. Pourcentage du $\mathrm{N}-\mathrm{NO}_{3}$ et du $\mathrm{P}$ total entrant piégé par la retenue.

Table.3. Percentage of the $\mathrm{N}-\mathrm{NO}_{3}$ and the total $\mathrm{P}$ entering trapped by the lake.

\begin{tabular}{ccccc}
\hline & $\begin{array}{c}\text { Apport annuel } \\
\text { de N-NO3 (t/an) }\end{array}$ & $\%$ N-NO3 piégé & $\begin{array}{c}\text { Apport annuel } \\
\text { de P total (t/an) }\end{array}$ & $\%$ P total piégé \\
\hline 1995 & 460 & $31.5 \%$ & 14.5 & $95.0 \%$ \\
1996 & 816 & $30.0 \%$ & 7.6 & $76.8 \%$ \\
1998 & 335 & $33.3 \%$ & 4.2 & $52.9 \%$ \\
\hline
\end{tabular}


Vioulou
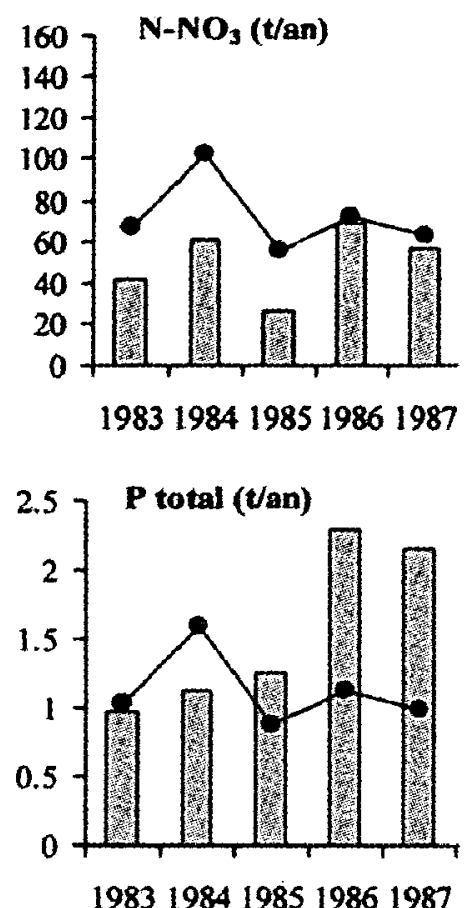

Rieutord

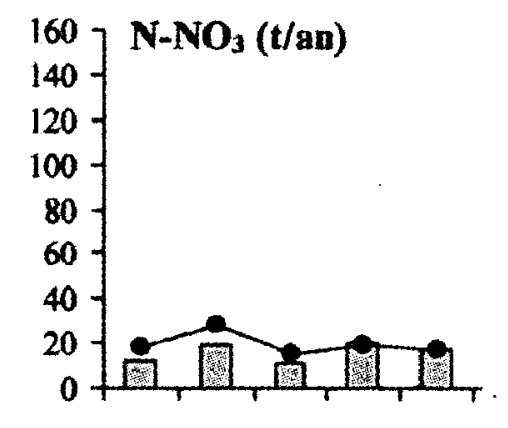

19831984198519861987

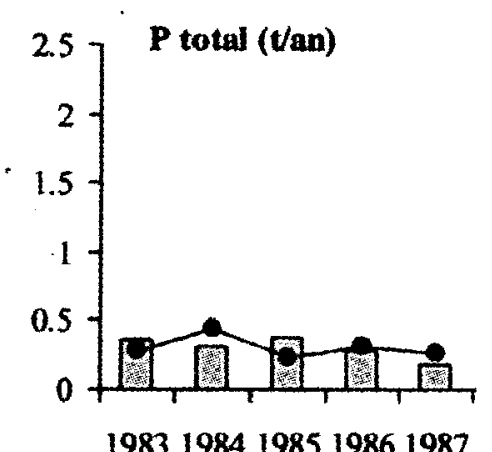

19831984198519861987

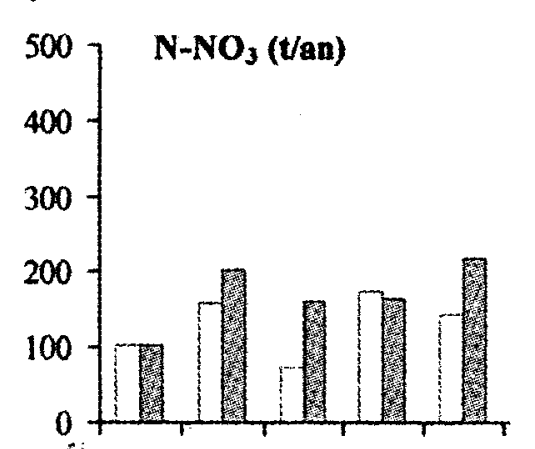

19831984198519861987

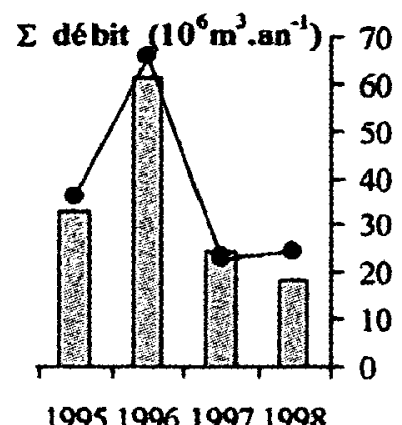

1995199619971998

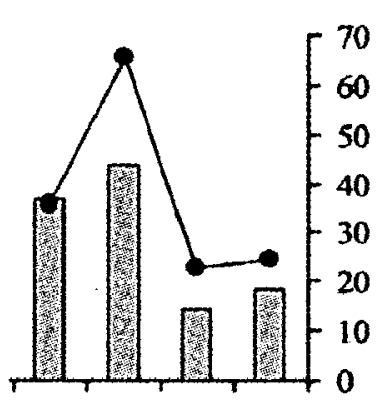

1995199619971998

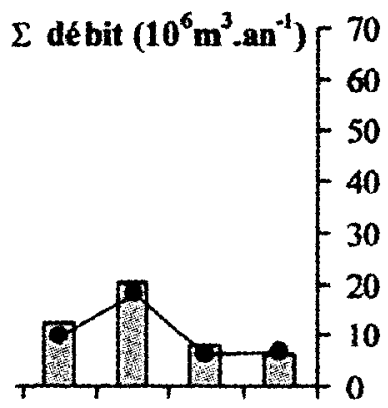

+ 1995199619971998

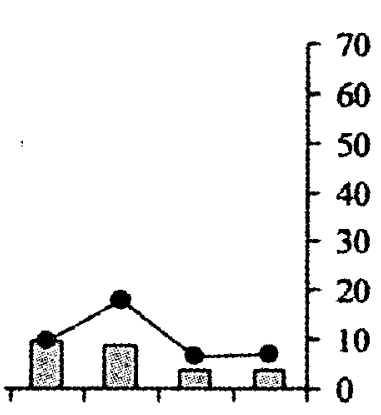

1995199619971998
Connes
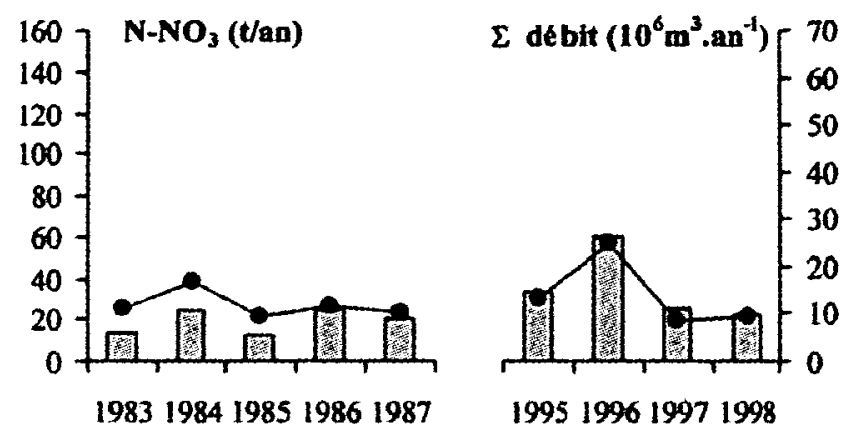

1995199619971998

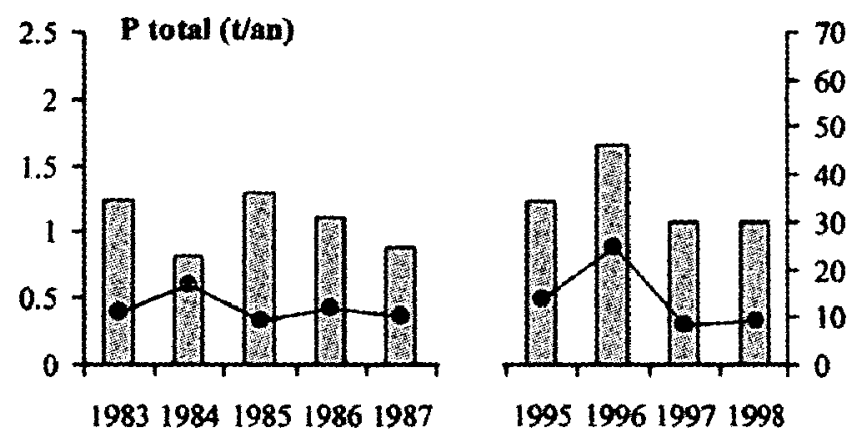

Bage
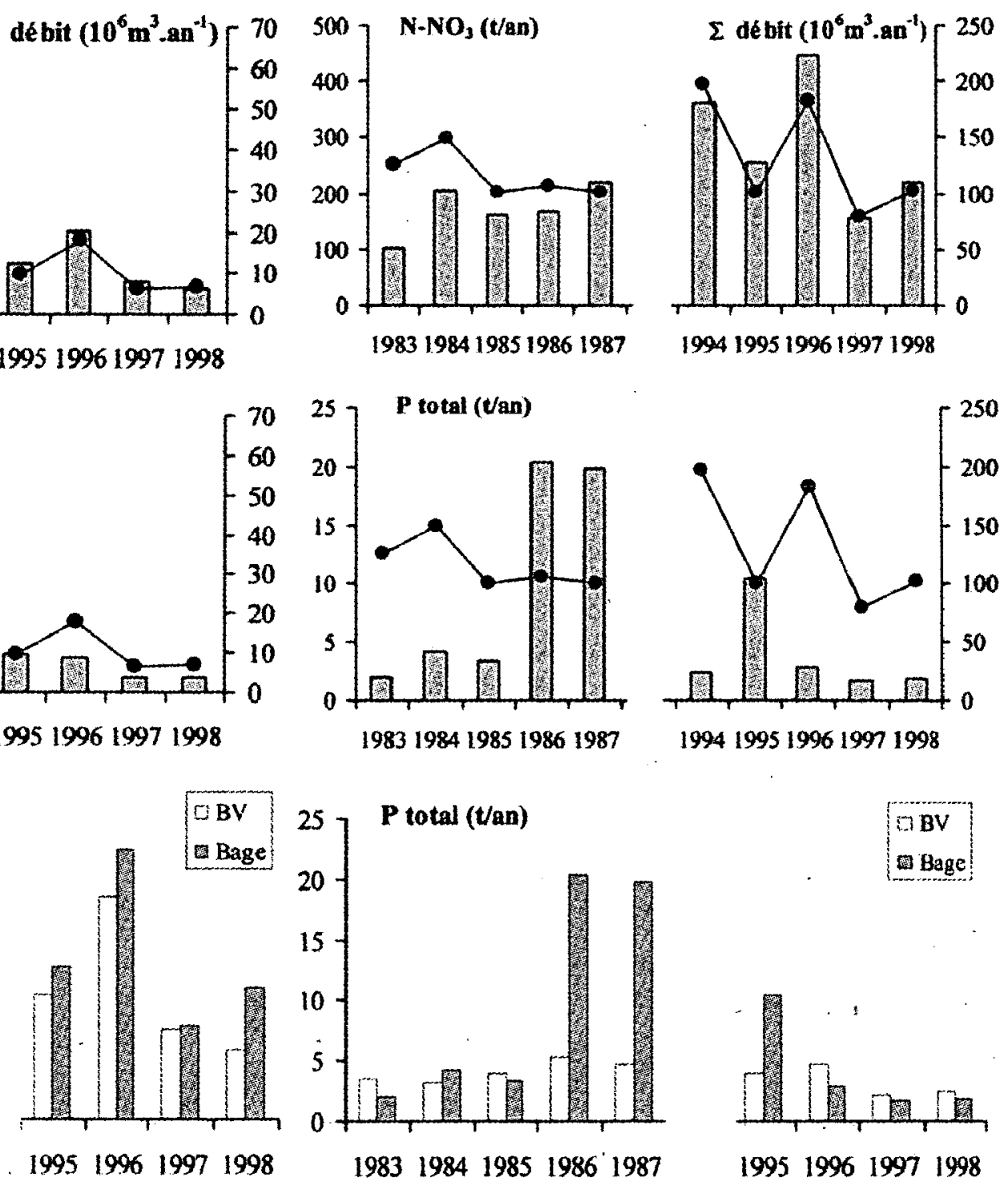

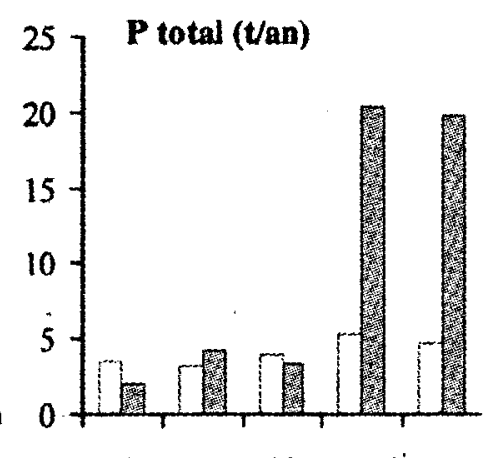

$1983198419851986^{\circ} 1987$

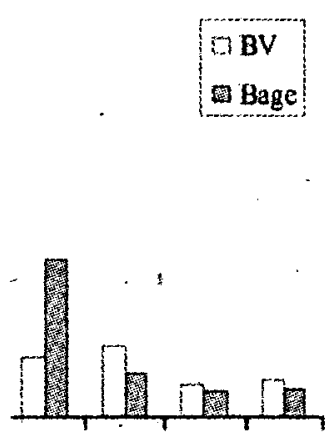

1995199619971998

Fig. 3. Flux annuels de $\mathrm{N}-\mathrm{NO}_{3}$ et $\mathrm{P}$ total dans les affluents et l'ensemble du bassin versant èt dans l'eau pompée à Bage ; débits annuels.

Fig. 3. Annual flows of $\mathrm{N}-\mathrm{NO}_{3}$ and total $\mathrm{P}$ from tributaries and the watershed and in water pumped from Bage ; annual flow rates. 
effectués dans la retenue de Pont de Salars, principalement parce que celle-ci alimente par pompage, à travers la retenue de Bage, les eaux de Pareloup (environ $50 \%$ des volumes entrant à Pareloup). Par conséquent, la qualité des eaux de Pareloup dépend de celles de Pont de Salars (Fig. 4, 5).

Les concentrations de $\mathrm{NO}_{3}$ dans le Viaur, affluent de la retenue de Pont de Salars sont, de 1994 à 1998, du même ordre de grandeur que celles des affluents de $\mathrm{Pa}$ reloup. Les concentrations en $P$ total sont également équivalentes à celles mesurées dans le Vioulou, excepté en 1995 et 1997, où elles sont beaucoup plus élevées, proches de celles mesurées dans le Connes. Les quantités de $\mathrm{NO}_{3}$ et de $\mathrm{P}$ total apportées varient de 120 à 330 tonnes par an de $\mathrm{N}-\mathrm{NO}_{3}$ et de 3 à 7 tonnes de $P$ total. Les concentrations maximales mesurées dans la retenue sont plus élevées qu'à Pareloup (+ $25 \%$ pour le NO3, plus du double pour le $\mathrm{P}$ total).

Bien qu'il n'y ait pas de développement massif de cyanobactéries, les concentrations maximales en chlorophylle sont plus élevées qu'à Pareloup (Tableau 4). L'évolution saisonnière de la chlorophylle ressemble à
Tableau 4. Concentrations maximales de chlorophylle a à Pareloup et Pont de Salars.

Table 4. Maximal concentrations of chlorophyll a into Pareloup and Pont de Salars.

\begin{tabular}{ccc}
\hline & \multicolumn{2}{c}{ Chlorophylle $a$} \\
& Pareloup & Pont de Salars \\
\hline 1994 & 9.2 & 12.9 \\
1995 & 13 & 21.4 \\
1996 & 11.5 & - \\
1997 & 7.8 & 16.5 \\
1998 & 8.9 & 30 \\
\hline
\end{tabular}

celle d'un lac eutrophe : concentration faible en hiver et au début du printemps et élevée en été. Le peuplement est lui aussi différent : parfois quelques cyanobactéries en hiver et au printemps, chlorophytes ou diatomées en été. Les différences interannuelles sont très marquées.

Le Secchi est peu profond (2,5 - 3 mètres), avec peu de variations saisonnières. Malgré son petit temps de
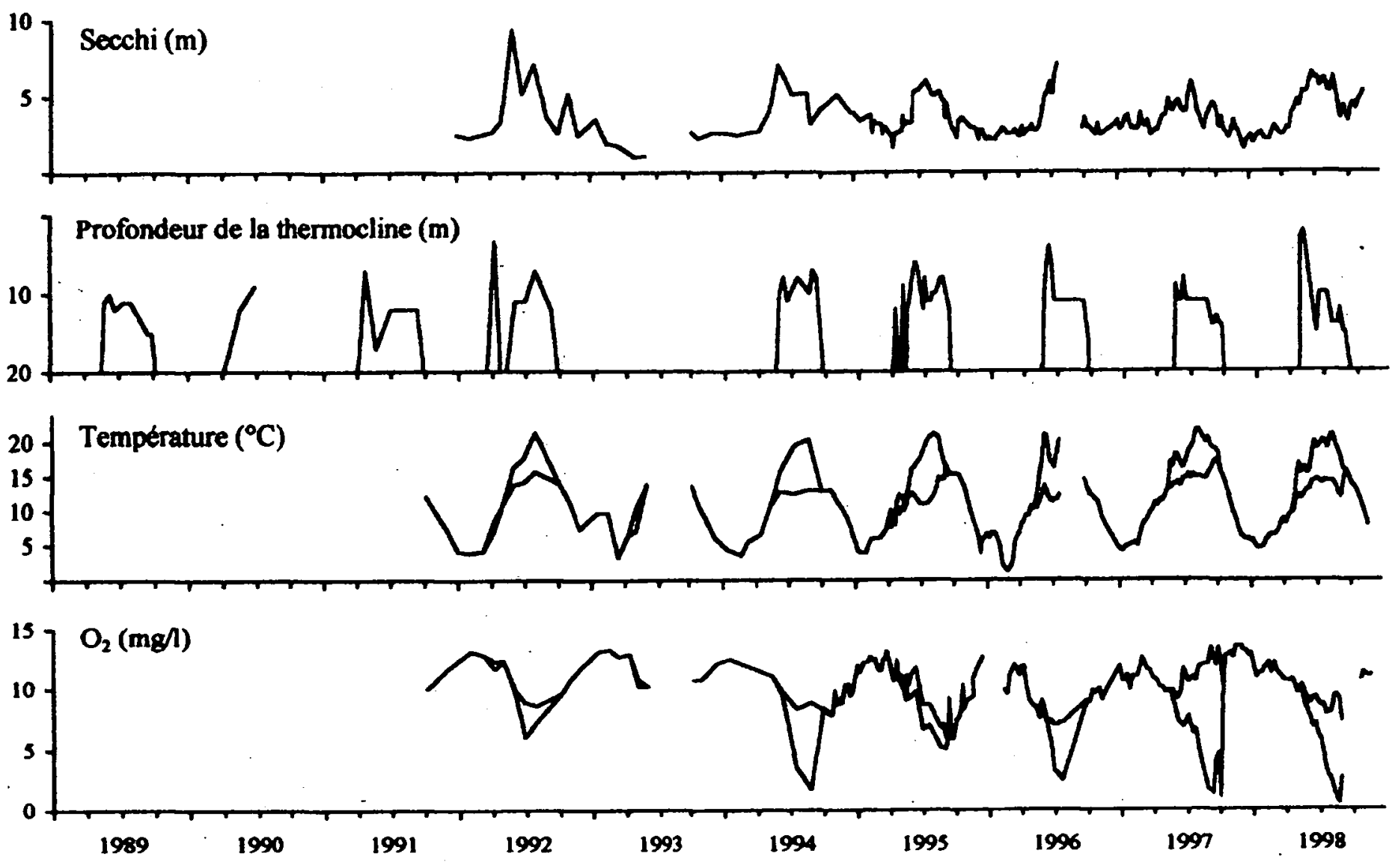

Fig. 4. Retenue de Pont de Salars : profondeur du disque de Secchi et de la thermocline ; température et oxygène dissous dans l'épi et l'hypolimnion.

Fig. 4. Lake Pont de Salars : Secchi disk depth and thermocline; temperature and dissolved oxygen in the epilimnion and hypolimnion. 

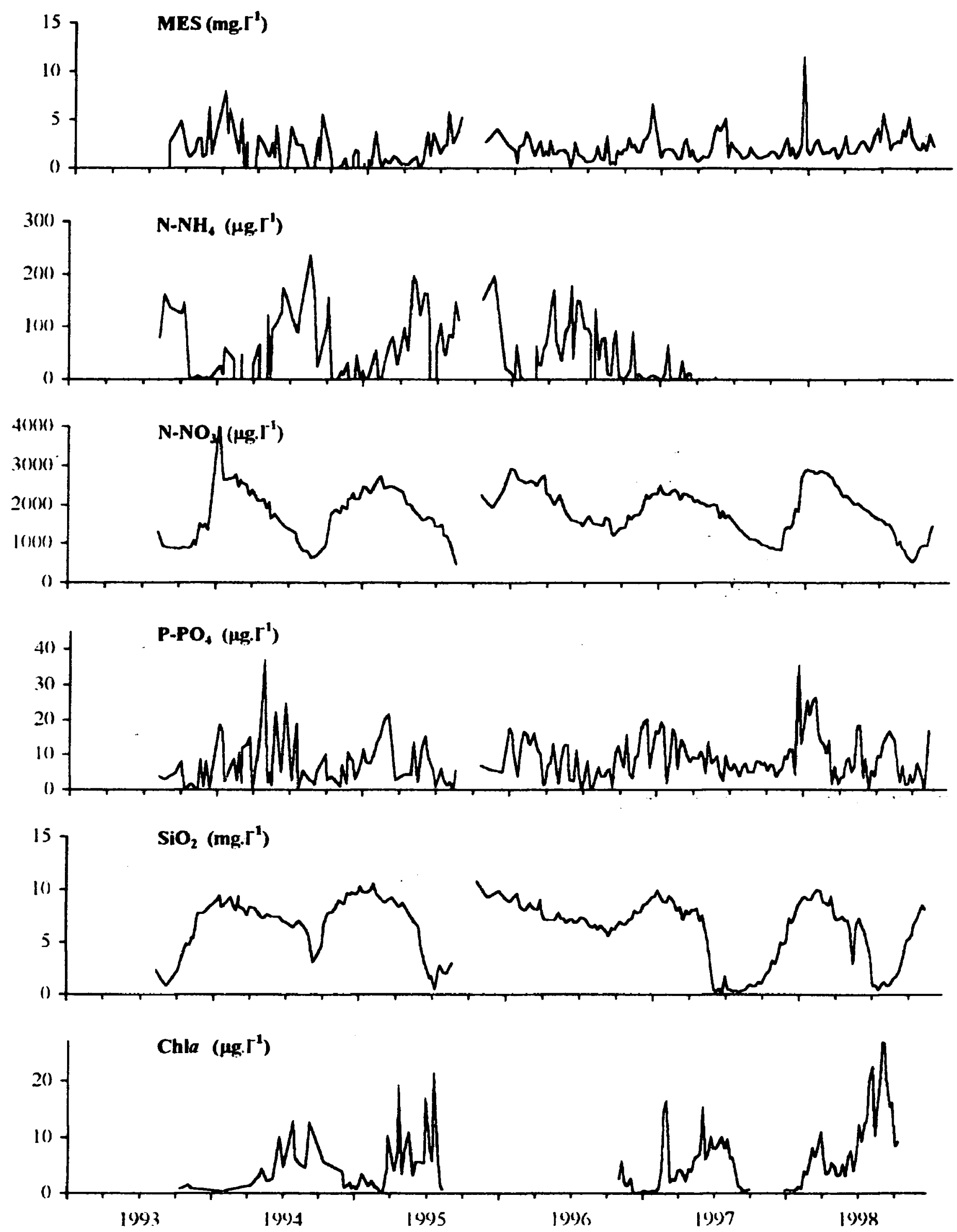

Fig. 5. Retenue de Pont de Salars : MES, nutriments et chlorophylle dans l'épilimnion.

Fig. 5. Lake Pont de Salars : MES (suspended solids), nutrients and chlorophyll a in the epilimnion. 
séjour (un mois en moyenne), la colonne d'eau est bien stratifiée en été et le déficit d' $\mathrm{O}_{2}$ dans l'hypolimnion est très marqué.

\section{La retenue de Pareloup}

\subsection{Evolution saisonnière des variables physico- chimiques (Fig. 6, 7,8)}

Profondeur du disque de Secchi : à part la période de la baisse des eaux en 1993 où il est très faible, le Secchi ne semble pas avoir été significativement perturbé depuis la remise en eau (comparaison faite avec la période 1978-1987 et l'année 1992) : 2 à 3 mètres durant le développement du phytoplancton printanier, environ 7 mètres durant la période stratifiée.

La profondeur de la thermocline, estimée d'après les relevés des profils thermiques, montre que la retenue est généralement bien stratifiée en été. En 1992, on observe une stratification précoce au début du mois d'avril, suivi d'un mélange profond début mai. En 1995 , la mise en place de la thermocline est agitée, les périodes de stratification et de mélange se succédant rapidement. Nous avons vu dans nos études précédentes (Salençon \& Thébault 1995, 1996, 1997), l'importance de telles situations pour la distribution des nutriments et, en particulier, la disponibilité de la silice pour le peuplement printanier de diatomées. En 1993, du fait de la baisse du plan d'eau au printemps, aucune véritable stratification n'a été observée.

Le calcul des concentrations moyennes dans l'épi et l'hypolimnion est pondéré par les volumes calculés avec la bathymétrie et la cote du plan d'eau. Cette représentation a été adoptée pour être utilisée par la suite comme référence à un modèle de simulation bicouche. La première valeur après vidange a été effectuée le 9/9/93, dans le cours naturel du Vioulou dans la retenue à sec, 8 jours avant la fermeture des vannes et le début de la remise en eau (17/9/93). La seconde mesure a été faite le 14/10/93, 4 semaines après la remise en eau.

Lorsque la stratification est bien marquée (1994, 1997 et 1998), on observe un déficit d' $O_{2}$ dans l'hypolimnion. A l'inverse, on n'observe pas d'anoxie en 1992 et 1995 (gradient thermique peu marqué en 1992 et mise en place chaotique de la thermocline saisonnière en 1995). Les matières en suspension (MES) n'ont été mesurées que depuis la vidange. Les concentrations se situent autour de valeurs variant de 0 à $5 \mathrm{mg} . \mathrm{l}^{-1}$. Les teneurs en azote ammoniacal $\left(\mathrm{N}-\mathrm{NH}_{4}\right)$ augmentent considérablement pendant la baisse du plan d'eau (relargage des sédiments et excrétion des poissons concentrés dans un faible volume) et reviennent à des valeurs faibles dès la fin de 1993 . Les valeurs manquantes en 1997 et 1998 sont dues à une mauvaise conservation des échantillons (analyse tardive). Les concentrations de nitrites $\left(\mathrm{N}-\mathrm{NO}_{3}\right)$ ne semblent pas présenter de cycles bien définis ni d'avoir été modifiées depuis la vidange. Les concentrations sont faibles $\left(25 \mu \mathrm{g} . \mathrm{l}^{-1}\right)$. La concentration des nitrates $\left(\mathrm{N}-\mathrm{NO}_{3}\right)$ a augmenté sensiblement après la vidange : $2800 \mu \mathrm{g} . \mathrm{l}^{-1}$ aux printemps 1994, 1996 et 1998, contre 2000-2200 de 1986 à 1992, et des concentrations maximales encore plus faibles $\left(1000-1200 \mu \mathrm{g} \cdot \mathrm{l}^{-1}\right)$ de 1983 à 1985 . Cela correspond à une augmentation sensible des concentrations depuis 1986 dans les affluents et aux apports de 1994 et 1996 (Fig. 3). Les concentrations d'orthophosphates $\left(\mathrm{P}^{-} \mathrm{PO}_{4}\right)$ varient de 0 à $10 \mu \mathrm{g} . \mathrm{l}^{-1}$ sans présenter de cycles. On observe deux valeurs légèrement plus élevées que la moyenne avant et après vidange, probablement liées à des échanges avec le sédiment. Le pic du printemps 1998 est lié au pompage dans une eau assez riche en $\mathrm{PO}_{4}$ à Bage/Pont de Salars $\left(25 \mu \mathrm{g} . \mathrm{l}^{-1}\right)$ mais n'a pas d'incidence sur le flux annuel. La silice, à cause de la perturbation subie par l'écosystème (peu de diatomées), n'a pas été limitante à la fin du printemps 1994, contrairement à toutes les autres années de l'étude. Les concentrations de silice dans la retenue ont presque doublé après la vidange : plus de 7.6 mg..$^{-1}$ en 1994 et 1996, 7 en 1995, 6.6 en 1998 contre des valeurs maximales variant de 3.5 à moins de $5 \mathrm{mg} . \mathrm{l}^{-1}$ de 1983 à la vidange.

On a d'abord pensé que cette augmentation était due au remplissage du lac par pompage dans les retenues de Bage-Pont de Salars, dont les eaux sont plus riches en silice que celles de Pareloup. Si cela avait été le cas, avec un temps de séjour du lac d'environ un an, les valeurs d'avant vidange auraient dû revenir rapidement à la normale. Ce qui n'est pas le cas. En fait, la quantité d'eau apportée par pompage pendant la phase de remplissage, du 16 septembre 1993 au 7 février 1994 (date de reprise du turbinage, cote 802.6), est de $54 \%$ des apports totaux (pompage + apports naturels). Cette proportion est celle que l'on constate toutes les autres années (50-60\% Fig. 1). Le remplissage a donc été fait avec le même mélange d'eaux que les autres années. Les concentrations dans les affluents n'ayant pas été modifiées après 1993 , la seule chose qui diffère est le volume des apports moyen annuels et donc les quantités de silice entrante (Fig. 8). Cela est particulièrement important en 1994 et 1996. L'augmentation des concentrations de silice, et dans une moindre mesure, celle des nitrates, $\mathbf{n}$ 'a donc pas la vidange pour origine, mais l'augmentation des apports. 


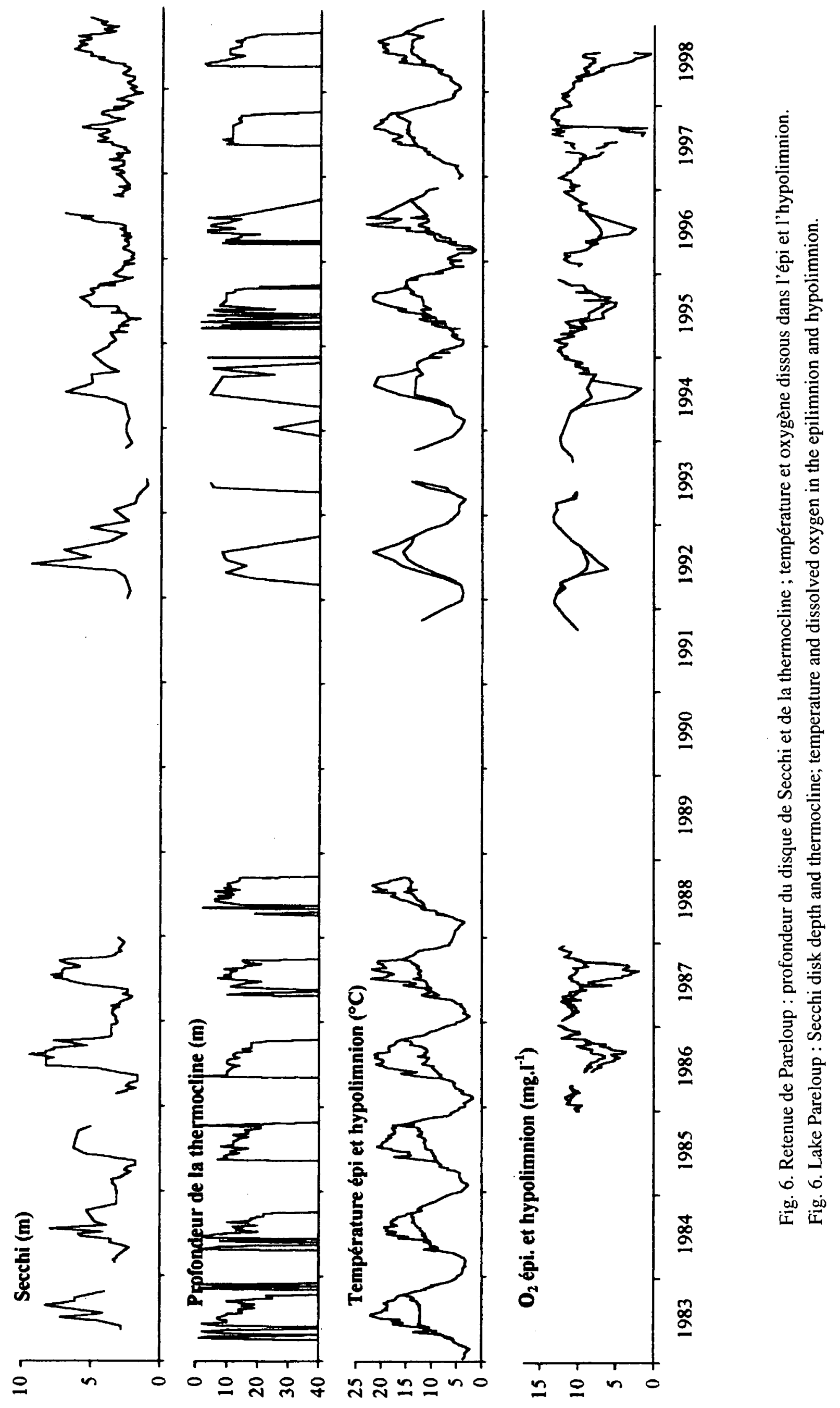



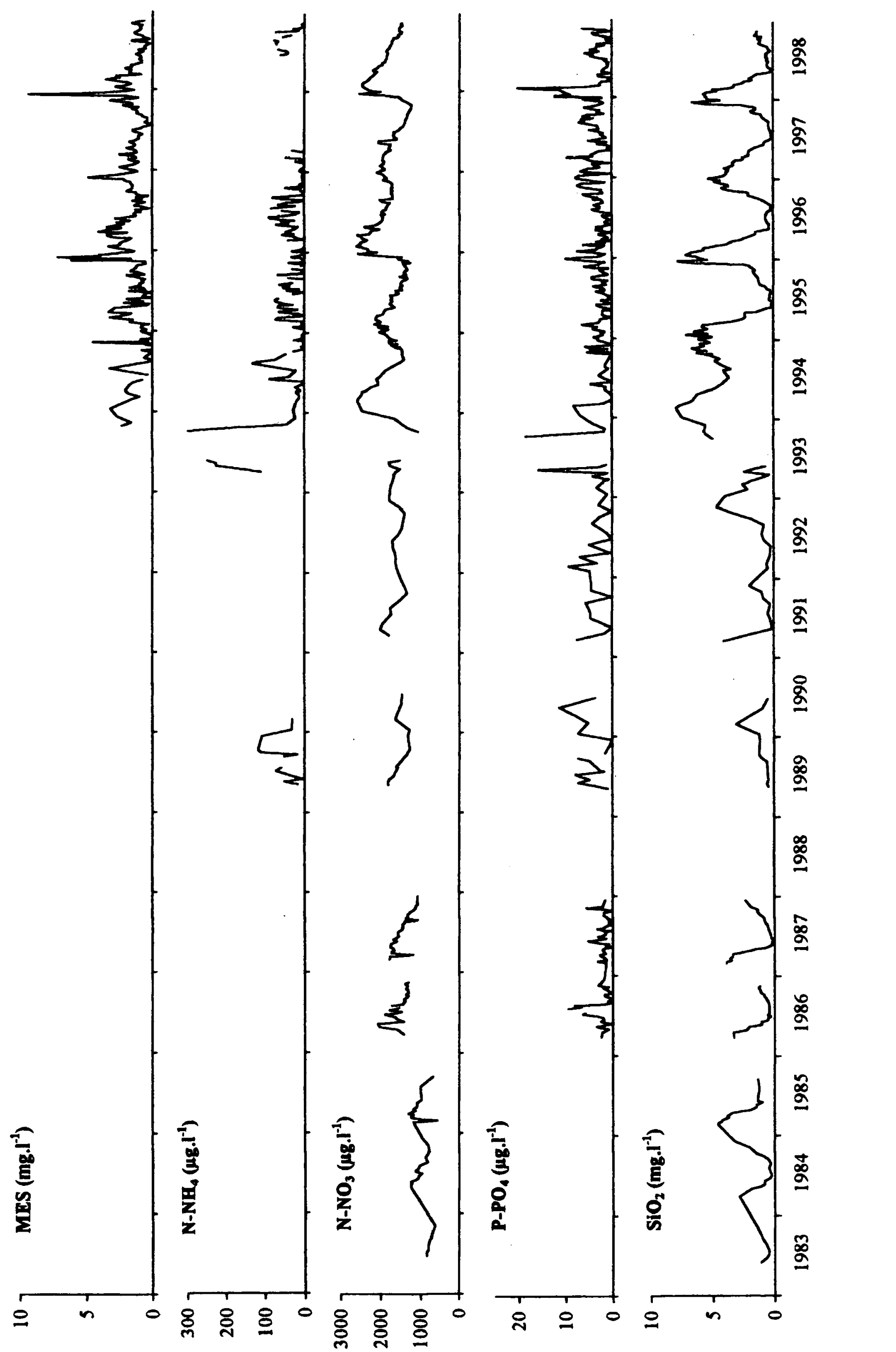

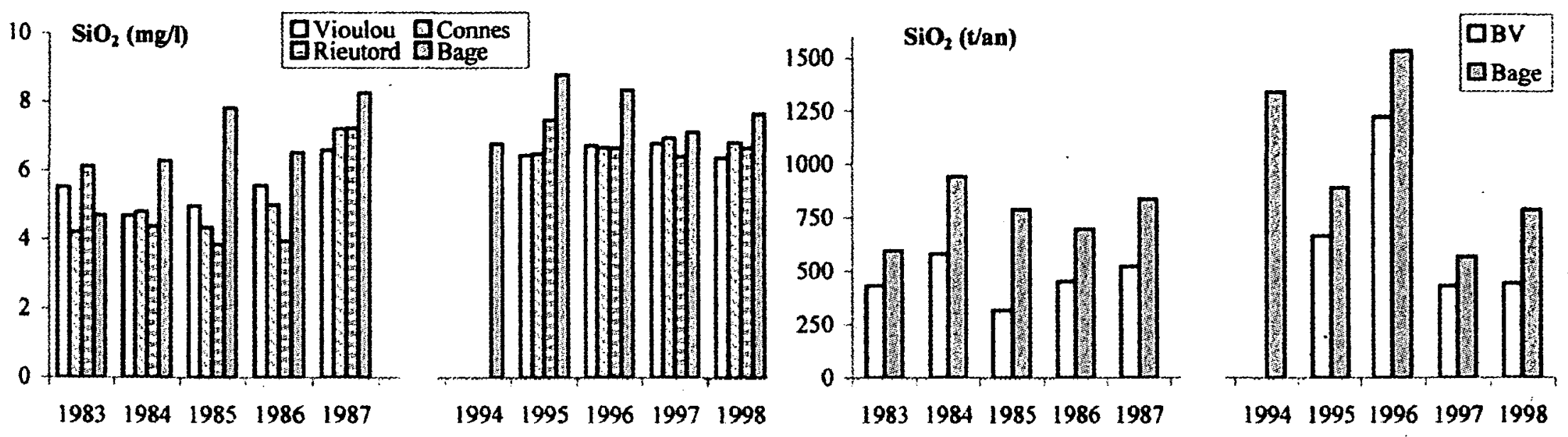

Fig. 8. Concentrations de silice dans les affluents et à Bage ; flux annuels pour l'ensemble du bassin versant et dans l'eau de Bage.

Fig. 8. Concentrations of silica in affluents and in Bage; Annual flows from the watershed and in the water pumped from Bage.

\subsection{Le phytoplancton (Fig. 9)}

— Les années pré-vidange 1986-1987 ; 1991-1992 : c'est le cycle classique déjà largement décrit pour les années 1983-1987 (Le Cohu et al. 1991, 1994, Thébault \& Salençon 1993, Salençon \& Thébault 1997) : développement à partir du début de l'hiver d'Aulacoseira ambigua (Grun.) Sim. et d'Aulacoseira subartica (O. Müll) Haworth. Ce sont les principales représentantes des diatomées centrales, consommées par certaines espèces du zooplancton, notamment les cyclopides adultes et les grands calanides (Einsle 1964, Infante \& Edmonson 1985). Asterionella formosa Hassal, représentant la quasi totalité des diatomées pennées, se développe à la fin de l'hiver et au printemps. On admet généralement que cette grande diatomée, compte tenu de sa forme, n'est pas consommée par le zooplancton. Certaines observations montrent cependant que Cyclops vicinus (présent à Pareloup) peut consommer A. formosa. On observe des différences interannuelles assez marquées dans l'abondance respective de ces deux diatomées. 1986, 1987 et 1991 sont caractérisées par une forte dominance d'A. formosa. En 1986, on observe des concentrations très élevés ; par contre, en 1987, les concentrations sont beaucoup plus faibles. Comme la quantité maximale de silice est un peu plus élevée que l'année précédente, et qu'elle a été entièrement consommée, il est fort probable que des petites centrales consommables (et consommées) se soient développées. En été, un phytoplancton diversifié et de petite taille se développe et est immédiatement consommé par le zooplancton ; à l'automne, on observe le développement de quelques diatomées et de Gonyostomum semen (Ehr.) Diesing (Le Cohu et al 1989). Il s'agit d'une raphydophycée, non consommée par le zooplancton. La biomasse du zooplancton brou- teur est maximale en été (de 45 à $90 \mathrm{mg}$ PS.1 ${ }^{-1}$ ), et présente des différences interannuelles aussi bien dans son amplitude que dans sa composition (Francisco \& Rey 1994).

- 1993, année de la vidange : abaissement du niveau en janvier et maintien du niveau autour de la cote 785 jusqu'à fin avril, soit $12 \%$ du volume et $35 \%$ de la surface. Les valeurs de Secchi sont beaucoup plus faibles qu'à l'ordinaire (1,5 $\mathrm{m}$ les deux derniers mois); cependant, les diatomées se développent abondamment et se maintiennent pratiquement jusqu'à l' «à sec» avec une très forte dominance d'Aulacoseira. La faible profondeur (donc une lumière moyenne dans la colonne d'eau plus élevée qu'à l'ordinaire) ainsi que l'absence de stratification qui permet à la silice de ne pas être limitante, favorise le développement des diatomées. Le zooplancton se développe bien au printemps, grâce aux grandes quantités de diatomées consommables (Fig. 10, Francisco 1999). Peu d'algues ont été comptées lors de la phase de remise en eau, cependant les quantités de chlorophylle mesurées sont assez importantes. Cela laisse supposer que du nanoplancton, difficile à détecter lors des comptages, ce soit développé durant cette période.

- Printemps 1994 : on observe très peu de diatomées, $10^{9} \mu \mathrm{m}^{3} .1^{-1}$ contre plus de $10 \cdot 10^{9} \mu \mathrm{m}^{3} .1^{-1}$ en temps normal. Les faibles valeurs de Chl $a$ et l'absence de consommation de silice en hiver et au début du printemps confirment qu'il $\mathrm{n}$ y a pas eu de diatomées à cette époque de l'année. Par contre, la consommation de silice dans la seconde partie du printemps (maijuin) laisse supposer que des diatomées, très rapidement consommées (ce qui explique les faibles concentrations), se soient développées. En effet, le zooplanc- 


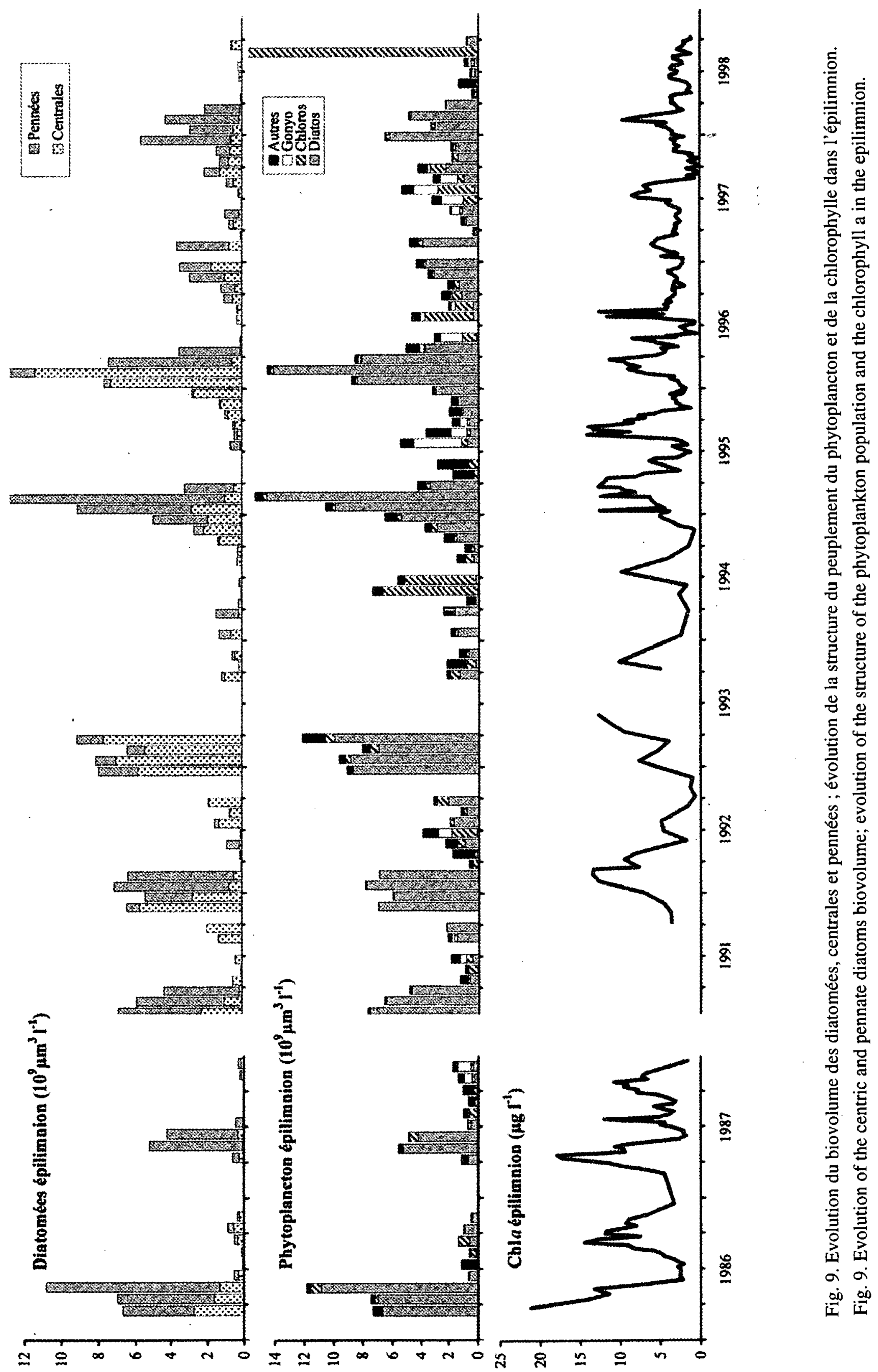




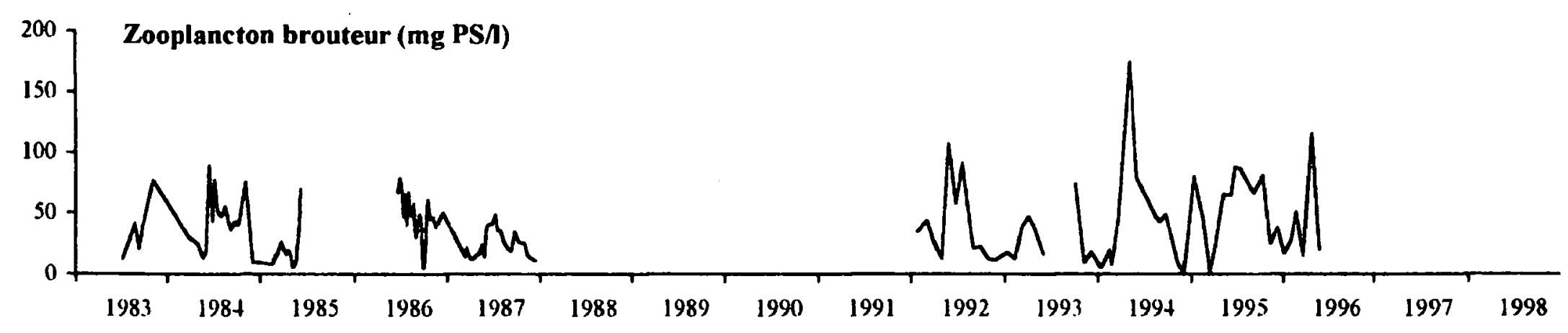

Fig. 10. Evolution du zooplancton brouteur.

Fig. 10. Evolution of the grazing zooplankton.

ton, dont l'abondance est très faible en hiver et au début du printemps croît rapidement pour atteindre des valeurs élevées pour cette saison (mi-mai), abondance favorisée par l'absence de poissons.

- En été, on observe un développement important et inhabituel dans sa composition et son importance (6 $10^{9} \mu \mathrm{m}^{3} \cdot \mathrm{l}^{-1}$ ) de chlorophycées (essentiellement Sphaerocystis $\mathrm{sp}$.), non-consommables par le zooplancton, d'où les concentrations élevées. La plupart de la production est consommée habituellement à cette époque.

- 1995 : les diatomées se développent abondamment dès le début de l'hiver et se maintiennent jusqu'à la fin du printemps épuisant complètement l'important stock de silice. Ce sont les centrales (principalement Aulacoseira) qui sont les plus précoces suivies par $A$. formosa, très largement dominante cette année là. Cependant la production d'Aulacoseira est certainement élevée compte tenu du développement inhabituel du zooplancton de janvier à fin mars $\left(80 \mu \mathrm{g} \mathrm{PS} . \mathrm{l}^{-1}\right)$. L'été, le microphytoplancton est consommé par le zooplancton dont la population croît abondamment $(>100 \mu \mathrm{g}$ PS. ${ }^{-1}$ ). Malgré le réempoissonnement débuté en 1994 et les poissons réfugiés dans les ruisseaux qui ont colonisé la retenue, le stock de poissons est encore petit par rapport à l'avant vidange, ce qui profite au zooplancton. A l'automne, on assiste au retour de $G$. semen, particulièrement abondant $\left(>610^{9} \mu \mathrm{m}^{3} \cdot \mathrm{l}^{-1}\right)$. Il était totalement absent en 1993 et 1994 ; il continuera à apparaître «normalement» chaque automne suivant.

- 1996 : compte tenu des teneurs élevées en silice, on observe une forte concentration de diatomées, comme en 1995, à la différence que Aulacoseira domine largement par rapport à $A$. formosa. Le zooplancton se développe peu par rapport aux deux années précédentes. Comme la nourriture est abondante, on peut imaginer que cela soit dû à une pression importante des poissons ; on observe en effet une forte densité d'alevins cette année là. Les comptages du zooplancton ont été arrêtés en juin.

— En 1997 et 1998, les concentrations de diatomées sont largement en deçà des années précédentes, y compris pré-vidange (inférieures à $410^{9} \mu \mathrm{m}^{3} . \mathrm{l}^{-1}$ et $\left.610^{9} \mu \mathrm{m}^{3} \cdot \mathrm{l}^{-1}\right)$. Les concentrations de silice ne semblent pas en cause, car même si elles sont moins importantes que les deux années précédentes, elles sont égales ou supérieures à celles des années pré-vidange. Compte tenu de la quantité de silice consommée, il est certain qu'il y a eu production de diatomées. Il est probable que celles-ci aient été ingérées par le zooplancton.

\section{Discussion}

Il est difficile de savoir exactement quelles sont les algues qui se développent car les comptages représentent une image instantanée de la partie restante, l'autre partie ayant été consommée. L'été est la saison où la production est la plus élevée et celle où la concentration de phytoplancton est la plus faible (Capblancq 1995, Thébault et al. 1999). Il est également difficile de tirer des conclusions à partir de l'analyse de l'abondance du zooplancton brouteur, lui-même consommé par les carnivores et les poissons planctonophages, dans des proportions mal connues. Seule la concentration des nutriments $\mathrm{NO}_{3}$ et $\mathrm{SiO}_{2}$ peut nous indiquer s'il y a eu production ; la complexité des mécanismes d'échange des différentes fractions du $\mathrm{P}_{-} \mathrm{PO}_{4}$, ne permet pas d'interpréter quoique ce soit pour ce nutriment (Thébault 1995).

Si les mesures de chlorophylle suivent assez bien celles des comptages (Fig. 9), on aperçoit parfois certaines dissemblances. Trois raisons à cela : 
- le rapport Chl $a$ : biovolume n'est pas constant. Par exemple, les Asterionella contiennent beaucoup plus de chlorophylle au début de l'hiver, quand la lumière est la plus faible, qu'à la fin du printemps (Le Cohu et al. 1991) ;

- si la chlorophylle mesurée est plus abondante que ce que laissent supposer les comptages (fréquemment en été), il s'agit, comme nous l'avons vu pour l'automne 1993, du nanoplancton qui n'a pas été détecté lors des comptages ;

- si la chlorophylle est moins abondante, il est possible que des échantillons n'aient pas été analysés à temps, d'où une sous-estimation des valeurs réelles de chlorophylle.

La particularité du lac de Pareloup est le développement abondant d'A. formosa au printemps. Etant peu ou pas consommée par le zooplancton, elle disparaît par sédimentation, ce qui immobilise dans le sédiment les nutriments qu'elle a consommés. Ceux-ci seront partiellement remis à disposition par relargage lors du mélange de la colonne d'eau à la fin de l'automne. Si certaines années, c'est principalement Aulacoseira qui se développe au détriment d'A. formosa, les nutriments assimilés se maintiennent dans la colonne d'eau par le biais du broutage et seront disponibles tout au long de l'été. Le rôle des diatomées printanières dans l'évolution de l'écosystème est donc capital. La figure 11 présente la relation entre le débit moyen journalier d'entrée (bassin versant + Bage) et le rapport pennées $(A$. formosa) : centrales (Aulacoseira) d'octobre à mai pour l'ensemble des années où nous disposons des comptages en biovolumes. Nous avons pu rajouter un point (année 1984-1985) à partir des données de Jrad (1989). On voit que lorsque les débits sont faibles, $\boldsymbol{A}$. formosa est fortement dominante. A l'inverse pour des débits élevés, la proportion d'Aulacoseira est beaucoup plus forte. Durant l'hiver 1992-1993, il n'y a pas eu d'entrée compte tenu de la vidange ; la retenue a donc subit d'importantes modifications hydrauliques et seules les diatomées centrales ont été capables de se développer. L'incapacité d'A. formosa à se développer dans des eaux trop courantes pourrait expliquer qu'on ne la rencontre pas dans des lacs à faible temps de séjour comme Pont de Salars.

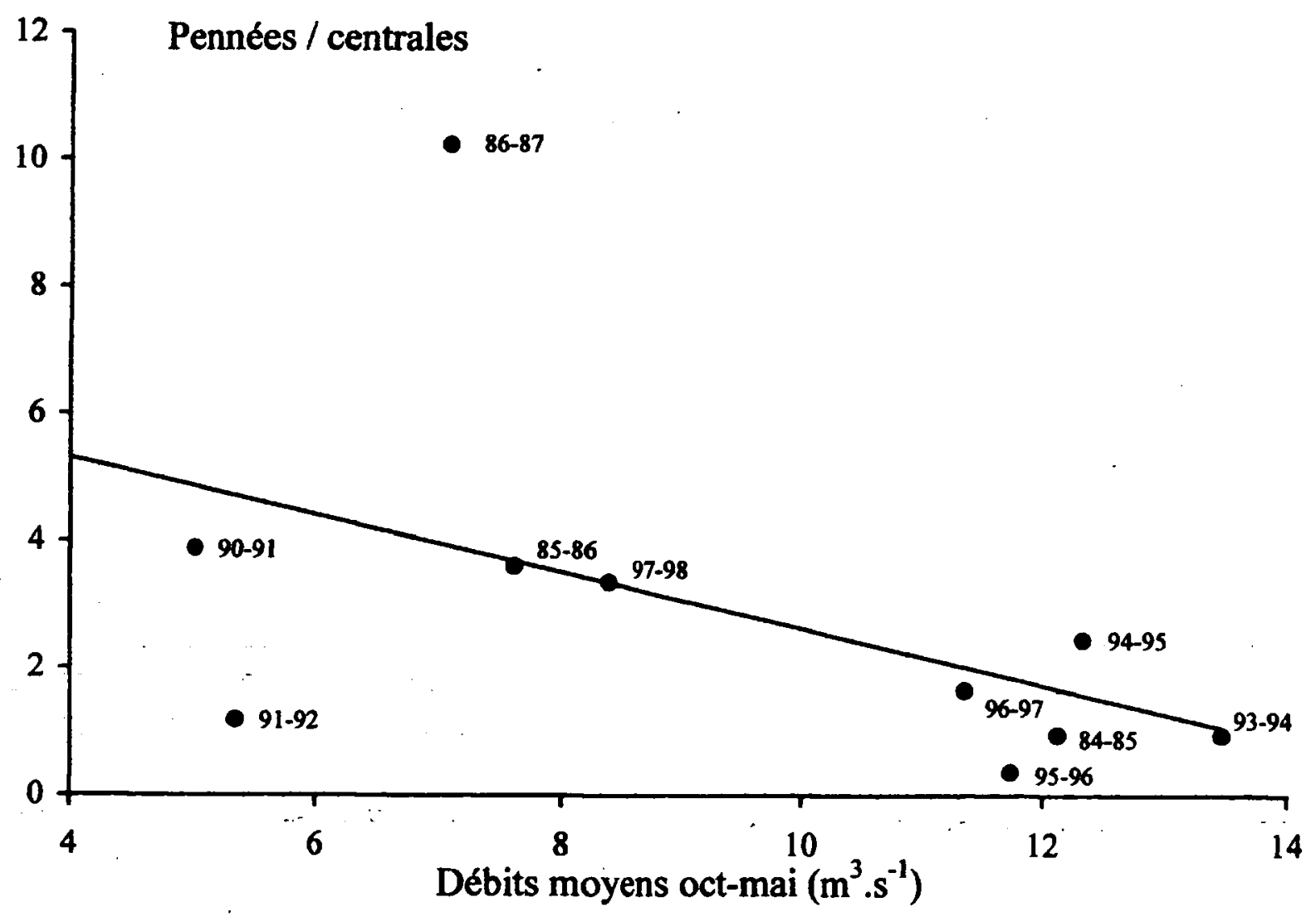

Fig .11. Relation entre le rapport diatomées pennées : diatomées centrales et le débit moyen journalier (bassin versant + Bage) d'octobre à mai.

Fig .11. Relation between the ratio pennate : centric diatoms and the daily average inflow (watershed $+\mathrm{Ba}-$ ge) from October to May. 


\section{Conclusion}

L'analyse de la période 1989-1998, avec les enseignements de celle qui avait été faite sur les mesures de 1983 à 1987, permettent de mieux appréhender l'écosystème. Une étude sur une longue période permet d'apprécier les différences interannuelles.

L'augmentation des concentrations de silice et dans une moindre mesure, de nitrates après la vidange, n'est pas lié, au remplissage à partir de Pont de Salars, mais à trois années pluvieuses consécutives qui ont eu pour conséquence de fortement augmenter les apports. Il apparaît qu'après la vidange, l'écosystème a été perturbé, entrainant en 1994 une situation n'ayant encore jamais été observée. L'écosystème a toutefois retrouvé un cycle typique dès 1995 , avec $A$. formosa au printemps et $G$. semen en automne. A. formosa n'est pas toujours dominante au printemps. Les forts débits, (ou petits temps de séjour) semblent favoriser la dominance d'Aulacoseira (consommée par le zooplancton) ce qui pourrait peut-être jouer un rôle important dans l'évolution de l'écosystème en été, par le biais du phosphore qui n'est pas piégé par sédimentation.

\section{Remerciements}

Ce travail a été réalisé dans le cadre d'une convention de recherche entre le Ministère de l'Environnement, Electricité de France (EDF) et le Centre National de la Recherche Scientifique pour la première phase, et dans le cadre d'un contrat de recherche entre EDF et l'Université Toulouse III représentée par le CESAC pour la seconde phase. Je remercie tous les membres de l'équipe Pareloup qui ont apporté leur contribution à cette étude pendant plus de 15 ans. Les analyses de chimie des eaux ont été réalisées par Mlle C. Mur depuis le début de l'étude ; la chlorophylle a été dosée par Mme N. Féroni les dernières années. Un grand merci à Marie-José Salençon pour ses conseils, pour le calcul des flux et de l'hydraulique. Une pensée particulièrement émue pour notre ami Jo Dagnac qui a quitté Pareloup pour le paradis...

\section{Travaux cités}

Capblancq J. 1995. — Production primaire autotrophe, In : R. Pourriot \& M. Meybeck (Eds), Limnologie Générale, Masson, Paris : 228-252.

Chung S.O. 2001. - Evolution du phytoplancton dans le réservoir de Pont de Salars. Suivi de la recolonisation après biomanipulation. Doctorat Université Paul Sabatier, Toulouse III, 170 p.

EDF Production Transport-Energie Midi-Pyrénées 1994. - Présentation de l'aménagement hydroélectrique du Pouget et du barrage de Pareloup. Hydroécol. Appl., 6 : 1-7.

Einsle U. 1964. - Die Gattung Cyclops s. str. im Bodensee. Arch. Hydrobiol., 64, 2 : 133-199.
Francisco P. 1999. - Dynamique de la communauté zooplanctonique et variations saisonnières de la composition biochimique (lipides, protéines) du plancton. Doctorat Université Paul Sabatier, Toulouse III : $219 \mathrm{p}$.

Francisco P. \& Rey J. 1994. - Etude du peuplement zooplanctonique de la retenue de Pareloup. Hydroécol. Appl., 6 : 175-196.

Horn W. 1981. - Phytoplankton losses due to zooplankton grazing in a drinking water reservoir. Int. Revue ges. Hydrobiol., 66 : 787-810.

Infante A. \& Edmonson W.T. 1985. - Edible phytoplankton and herbivorous zooplankton in Lake Washington. Arch. Hydrobiol. Beih. Ergebn. Limnol., 21 : 161-171.

Jrad A. 1989. - Phytoplancton et production primaire dans le lac de Pareloup. Thèse de $3^{\text {ème }}$ cycle, Université Paul Sabatier, Toulouse III : $166 \mathrm{p}$.

Le Cohu R., Guitard J., Comoy N. \& Brabet J. 1989. - Gonyostomum semen (Raphidophycées), nuisance potentielle des grands réservoirs français ? L'exemple du lac de Pareloup. Arch. Hydrobiol., $117: 225-236$.

Le Cohu R., Comoy N., Guitard J. \& Brabet J. 1991. — Périodicité du phytoplancton dans un réservoir de moyenne profondeur : le lac de Pareloup (Massif Central, France), un exemple de succession cyclique. Ann. Limnol., 27 : 197-214.

Le Cohu R., Brabet J., Guitard J. \& Comoy N. 1994. - Evolution temporelle et spatiale du phytoplancton dans le réservoir de Pareloup. Hydroécol. Appl., 6:139-151.

Merle G., Mosnier D. \& Toureng J.N. 1994. - La vidange de la retenue de Pareloup en 1993 : une étape-clef dans la vie du lac. $H y$ droécol. Appl., $6:$ 427-446.

Moatar F. \& Meybeck M., 2002. - Reliability of nutrient loads data in the Loire river. Hydrological Processes, (in prep.).

Salençon M.J. \& Thébault J.M. 1995. - Modélisation de l'écosystème du Lac de Pareloup (Aveyron), In : R. Pourriot \& M. Meybeck (Eds), Limnologie Générale, Masson, Paris :765-805.

Salençon M.J. \& Thébault J.M. 1996. — Simulation model of a mesotrophic reservoir (Lac de Pareloup) : MELODIA, an ecosystem reservoir management model. Ecol. Modelling, 84 : 163-187.

Salençon M.J. \& Thébault J.M. 1997. — Modélisation d'écosystème lacustre. Application à la retenue de Pareloup (Aveyron). Masson, Paris : $200 \mathrm{p}$.

Salençon M.J., Thébault J.M. \& Capblancq J. 1989. — Etude de la retenue de Pareloup. Bilan des travaux réalisés en 1988 dans le cadre de la Convention EDF-Ministère de l'Environnement. Rapport HE31/89-13, Electricité de France, Paris : 77 p.

Sivadier F., Thébault J.M. \& Salençon M.J. 1994. - Bilan du phosphore total dans la retenue de Pareloup. Hydroécol. Appl., 6 : 115-138.

Thébault J.M. 1995. - Representation of phosphorus in lake ecosystem models. Ann. Limnol., 31 : 143-149.

Thébault J.M., Capblancq J. \& Petit M. 1999. — Influence de la morphométrie et du brassage vertical sur la production primaire nette du phytoplancton. C.R. Acad. Sci. Paris, 322:63-70.

Thébault J.M. \& Salençon M.J. 1993. - Simulation model of a mesotrophic reservoir (Lac de Pareloup) : biological model. Ecol. Modelling, $65: 1-30$. 